\title{
Sudden cardiac death is associated both with epilepsy and with use of antiepileptic medications
}

\author{
Abdennasser Bardai, ${ }_{1}^{1,2,3}$ Marieke T Blom, ${ }_{1}^{1}$ Charlotte van Noord, ${ }_{1}^{3,4}$ \\ Katia M Verhamme, ${ }^{3}$ Miriam C J M Sturkenboom, ${ }^{3,5}$ Hanno L Tan ${ }^{1}$
}

${ }^{1}$ Department of Cardiology, Heart Center, Academic Medical Center, University of Amsterdam, Amsterdam, The Netherlands ${ }^{2}$ Interuniversity Cardiology Institute Netherlands, Utrecht, The Netherlands

${ }^{3}$ Department of Epidemiology, Erasmus Medical Center, Rotterdam, The Netherlands ${ }^{4}$ Department of Internal Medicine, Erasmus Medical Center, Rotterdam,

The Netherlands

${ }^{5}$ Department of Medical Informatics, Erasmus Medical Center, Rotterdam,

The Netherlands

\section{Correspondence to}

Dr Hanno L Tan, Department of Cardiology, Academic

Medical Center, Meibergdreef 9, Amsterdam 1105 AZ, The Netherlands;

h.l.tan@amc.nl

Received 6 February 2014 Revised 6 June 2014 Accepted 27 June 2014 Published Online First 16 July 2014

\section{ABSTRACT \\ Objective Epilepsy is associated with increased risk for sudden cardiac death (SCD). We aimed to establish, in a community based study, whether this association is mediated by epilepsy per se, use of antiepileptic medications (AEMs), or both.}

Methods We studied SCD cases and age/sex matched controls in a case-control study in a large scale general practitioners' research database ( $n=478661$ patients). SCD risk for symptomatic epilepsy (seizure $<2$ years before SCD), stable epilepsy (no seizure $<2$ years before SCD), and use of AEMs (any indication) was determined. Results We identified 926 SCD cases and 9832 controls. Fourteen cases had epilepsy. Epilepsy was associated with an increased SCD risk (cases 1.5\%, controls $0.5 \%$; adjusted OR 2.8, 95\% Cl 1.4 to 5.3 ). SCD risk was increased for symptomatic epilepsy (cases $0.9 \%$, controls $0.1 \%$; adjusted OR 5.8, 95\% Cl 2.1 to 15.6), but not with stable epilepsy (cases $0.6 \%$, controls $0.4 \%$; adjusted OR $1.6,95 \% \mathrm{Cl} 0.7$ to 4.1 ). AEM use was found in 23 cases and was associated with an increased SCD risk (cases 2.5\%, controls $0.8 \%$; adjusted OR overall $2.6,95 \% \mathrm{Cl} 1.5$ to 4.3 ) among symptomatic epilepsy cases (cases $0.9 \%$, controls $0.1 \%$; adjusted OR $6.4,95 \% \mathrm{Cl} 2.4$ to 17.4 ) and non-epilepsy cases (cases $1.0 \%$, controls $0.4 \%$; adjusted OR 2.3, 95\% Cl 1.01 to 5.2). Increased SCD risk was associated with sodium channel blocking AEMs (cases 1.6\%, controls $0.4 \%$; adjusted OR 2.8, 95\% Cl 1.1 to 7.2 ), but not with non-sodium channel blocking AEMs. Carbamazepine and gabapentin were associated with increased SCD risk (carbamazepine: cases 1.1\%, controls 0.3\%; adjusted OR 3.2, 95\% Cl 1.1 to 9.2; gabapentin: cases $0.3 \%$, controls $0.1 \%$; adjusted OR $5.7,95 \% \mathrm{Cl} 1.2$ to 27.9 ).

Conclusions Epilepsy and AEM use are both associated with increased SCD risk in the general population. Poor seizure control contributes to increased SCD risk in epilepsy, while sodium channel blockade contributes to SCD susceptibility in AEM users.

\section{INTRODUCTION}

Sudden cardiac death (SCD) accounts for 50\% of cardiovascular deaths in Western societies. Its causes are complex. ${ }^{1}$ Part of SCD is explained by established risk factors such as heart failure and ischaemic heart disease. $^{2}$ SCD risk may also be increased by drugs, including those used to treat non-cardiac disease, because these drugs impair cardiac repolarisation (QT prolongation) or depolarisation (QRS widening), thereby increasing the risk for fatal cardiac arrhythmias (ventricular fibrillation (VF)). Such non- cardiac drugs are associated with QT prolongation, QRS widening, and SCD. ${ }^{3-5}$

Similarly, it is increasingly recognised that noncardiac disease may be associated with increased SCD risk, possibly by affecting cardiac electrophysiology. Epilepsy, in particular, is associated with increased risk for sudden death. Sudden death is a leading cause of death in epilepsy, especially among patients with poorly controlled epilepsy and recurrent seizures. $^{6}$ Lethal cardiac arrhythmias are potential causes for sudden death in epilepsy. ${ }^{7}$ In a recent systematic ECG based study, we provided the first proof that epilepsy is associated with a threefold increased risk for VF and SCD in the general population. ${ }^{8}$ The causes for this association are unresolved. Two mechanisms may play a role. First, epilepsy per se may increase SCD risk. Case reports of patients with poorly controlled epilepsy in whom sudden death was observed support this notion. ${ }^{6}$ Secondly, use of antiepileptic medications (AEMs) may cause cardiac arrhythmias, as some AEMs may impede cardiac depolarisation by blocking cardiac sodium channels; this mechanism can evoke lethal arrhythmias in susceptible individuals (QT prolongation by AEMs has not been reported). ${ }^{9}$

So far, these potential mechanisms have not been systematically studied in a community based study. Our aim was to estimate the effects of epilepsy and AEM use on SCD risk. To probe the role of epilepsy, we studied whether SCD risk is more elevated in patients with severe epilepsy than in patients with non-severe epilepsy. To probe the role of AEMs, we studied whether SCD risk is increased in non-epilepsy patients who use AEMs (eg, for neuralgia), and whether SCD risk is more elevated in AEMs with sodium channel blocking properties.

\section{METHODS}

\section{Setting and study design}

This investigation was a case-control study conducted in conjunction with the Integrated Primary Care Information (IPCI) project. IPCI is a longitudinal observational database that contains the complete medical records of 478661 patients from a large group of general practitioners (GPs) in the Netherlands. In the Dutch healthcare system, the GP plays a pivotal role by acting as a gatekeeper for all medical care. Details of the database have been described elsewhere ${ }^{10} 11$ Briefly, IPCI contains computer based records, including coded and anonymous data on patient demographics, symptoms (in free text), and diagnoses (using the International
To cite: Bardai $A$, Blom MT, 2015;101:17-22. 
Classification for Primary Care (ICPC) and free text) from GPs and specialists. ${ }^{12}$

The source population comprised all patients aged $\geq 18$ years in the IPCI database with a valid database history (date of registration with GP) of at least 1 year. The study population comprised all SCD (see below for SCD definition) cases plus their controls in the source population. For each case of SCD, up to 20 controls were randomly drawn from the source population (incidence density sampling) matched by age (year of birth), gender, and SCD date.

\section{Definition of SCD}

SCD in IPCI was defined according to the European Society of Cardiology guidelines: (1) witnessed natural death, heralded by abrupt loss of consciousness, within $1 \mathrm{~h}$ of onset of acute symptoms; or (2) unwitnessed, unexpected death of someone seen in a stable medical condition $<24 \mathrm{~h}$ previously with no evidence of a non-cardiac cause. ${ }^{13}$ Medical and demographic data were screened for all deaths in the database. The medical records of all individuals who died were reviewed manually to assess whether death could be classified as SCD. Individuals were classified as SCD cases if the medical record indicated that death occurred within $1 \mathrm{~h}$ after onset of cardiovascular symptoms and if the following wording was found in the free text: 'SCD', 'acute cardiac death', 'mors subita', 'sudden death', 'died suddenly', 'died unexpectedly' or if this was an unwitnessed, unexpected death of someone seen in 'good health' or in stable medical condition $<24 \mathrm{~h}$ previously. Individuals with evidence of a non-cardiac cause (eg, suicides, pneumonia, convulsion, choking, or cerebrovascular accident) were not classified as SCD. Validation of SCD was performed independently by two medically trained persons blinded to exposure. A third expert arbitrated in case of a discrepancy.

\section{Use of AEMs}

All available AEMs in the Netherlands were studied: phenytoin, carbamazepine, levetiracetam, vigabatrin, ethosuximide, clonazepam, phenobarbital, primidone, lamotrigine, oxcarbazepine, gabapentin, and valproic acid. AEMs were classified into two groups based on their published ability to block the sodium channel (neural and/or cardiac). Sodium channel blocking drugs included: phenytoin, carbamazepine, oxcarbazepine, lamotrigine, and gabapentin. ${ }^{14-19}$

The duration of each prescription was calculated by dividing the total number of units issued per prescription by the prescribed daily number of units. AEM use was defined as current if the SCD date fell within a period of use or a maximum of 28 days after the end of the last prescription (to deal with carryover and non-compliance effects). Past use was defined as discontinuation of an AEM > 28 days before the date of the SCD. If patients had no prescription for an AEM before the SCD date, they were considered as non-exposed.

\section{Definition of epilepsy}

All GP records of cases and controls using an AEM were reviewed manually to confirm a diagnosis of epilepsy. Patients were categorised as having epilepsy if the ICPC code for epilepsy was recorded in the GP record and/or epilepsy could be derived from the GP record (eg, seizures and use of AEMs, citation in the GP record of communications between GP and neurologist confirming epilepsy or long term use of AEMs with exclusion of other diagnoses for which AEMs could be used). Only cases and controls with a diagnosis of epilepsy and current treatment with AEMs were classified as epilepsy. Patients were defined as having stable epilepsy if they were seizure-free in the 2 years before the SCD date, or as having symptomatic epilepsy if they had at least one seizure in the 2 years before the SCD date, either because they were therapy resistant or because they had epilepsy de novo. ${ }^{20}$

\section{Analysis of non-epilepsy patients using AEMs}

All GP records of cases and controls of patients who used an AEM, but had no epilepsy, were reviewed manually to retrieve the indication for AEM prescription (mainly chronic postherpetic neuralgia, chronic diabetic neuropathic pain, or restless legs).

\section{Covariates}

Known risk factors and other covariates for SCD were gathered from the medical records through computerised searches and manual validation. Myocardial infarction, transient ischaemic attack, stroke, arrhythmia, and heart failure were assessed, based on the diagnoses provided by GP and medical specialists in the medical records. ${ }^{21}$ Use of QTc prolonging drugs, antiarrhythmic drugs, digoxin, diuretics, calcium channel blockers, and $\beta$-blockers at the index date were considered covariates.

\section{Statistical analysis}

The relative risk of SCD associated with AEM use was estimated by calculation of the adjusted ORs using conditional logistic regression analysis. Clinically relevant covariates that were univariately associated with SCD (at a level of $\mathrm{p}<0.1$ ) were included in the regression analyses if they changed the point estimate of the association between AEM use and SCD by $>5 \%$. $^{22}$ To study whether epilepsy was associated with increased SCD risk, we classified patients as having stable or symptomatic epilepsy and analysed this specifically. To study whether AEM use was associated with increased SCD risk, we examined SCD risk for patients with or without epilepsy. Among current users, we evaluated the effect of the duration of use, defined as the time between first intake and the index date. We investigated potential effect modification by age and gender. All analyses were performed using SPSS for Windows V.20.0 (Chicago, Illinois, USA).

\section{RESULTS}

\section{Subject characteristics}

The source population comprised 478661 subjects. During a follow-up of 1905382 person-years, 14259 persons died, including 926 SCD cases. We identified 9832 controls. The mean age of cases was 71.7 years and $62.0 \%$ were male (table 1).

\section{Association between epilepsy and SCD}

Fourteen cases $(1.6 \%)$ and 49 controls $(0.5 \%)$ had a diagnosis of epilepsy. Epilepsy was associated with an almost threefold increased risk for SCD (cases $1.5 \%$, controls $0.5 \%$; OR adjusted $2.8,95 \%$ CI 1.4 to 5.3 ) (table 2). Patients with stable epilepsy had no increased SCD risk (cases $0.6 \%$, controls $0.4 \%$; $\mathrm{OR}_{\text {adjusted }} 1.6,95 \% \mathrm{CI} 0.7$ to 4.1 ) (table 2), while patients with symptomatic epilepsy had an almost sixfold increased SCD risk (cases $0.9 \%$, controls $0.1 \%$; OR adjusted $5.8,95 \%$ CI 2.1 to 15.6 ) (table 2). In the symptomatic epilepsy group, seven patients had therapy resistant epilepsy, and one epilepsy de novo 4.5 months before SCD. The last seizure occurred 20 months ( $n=1$ patient), 11 months $(n=1)$ or within 5 months $(n=6)$ before SCD, while median seizure frequency was 2.5 per 2 years. There was no statistically significant difference in age or sex between patients with stable epilepsy and patients with symptomatic epilepsy 
Table 1 Baseline characteristics, demographics, and distribution of covariates

\begin{tabular}{|c|c|c|c|}
\hline Characteristic & $\begin{array}{l}\text { SCD } \\
\text { cases } \\
n=926\end{array}$ & $\begin{array}{l}\text { Controls } \\
n=9832\end{array}$ & $\mathrm{OR}^{*}(95 \% \mathrm{Cl})$ \\
\hline \multicolumn{4}{|l|}{ Gender } \\
\hline Male & $574(62.0)$ & $6319(64.3)$ & \\
\hline Female & $352(38.0)$ & $3513(35.7)$ & \\
\hline Age in years, mean (SD) & $71.7(13.7)$ & $66.1(13.6)$ & \\
\hline$\leq 55$ & $121(13.1)$ & $2145(21.8)$ & \\
\hline $55-65$ & $145(15.7)$ & $2147(21.8)$ & \\
\hline $66-75$ & $248(26.8)$ & $2818(28.7)$ & \\
\hline$>75$ & $412(44.5)$ & $2722(27.7)$ & \\
\hline \multicolumn{4}{|l|}{ Comorbidities } \\
\hline $\begin{array}{l}\text { Ischaemic cerebrovascular or } \\
\text { cardiovascular disease }\end{array}$ & $273(29.5)$ & $1448(14.7)$ & $2.0(1.7$ to 2.3$)$ \\
\hline Arrhythmia & $83(9.0)$ & $553(5.6)$ & $1.3(1.0$ to 1.7$)$ \\
\hline Hypertension & $364(39.3)$ & 3005 (30.6) & $1.3(1.1$ to 1.5$)$ \\
\hline Diabetes mellitus & $169(18.3)$ & $833(8.5)$ & 2.3 (1.9 to 2.8$)$ \\
\hline Heart failure & $162(17.5)$ & $397(4.0)$ & 4.0 (3.2 to 5.0$)$ \\
\hline Hypercholesterolaemia & $137(14.8)$ & $1085(11.0)$ & $1.7(1.3$ to 2.0$)$ \\
\hline Smoking & $197(21.3)$ & 2024 (20.6) & $1.3(1.1$ to 1.5$)$ \\
\hline Alcohol abuse & $22(2.4)$ & $84(0.9)$ & $3.5(2.1$ to 5.9$)$ \\
\hline \multicolumn{4}{|l|}{ Concomitant medication } \\
\hline QT prolonging drugs & $50(5.4)$ & $188(1.9)$ & $1.7(1.2$ to 2.4$)$ \\
\hline Antiarrhythmic drugs & $15(1.6)$ & $76(0.8)$ & $1.9(1.0$ to 3.5$)$ \\
\hline Digoxin & $76(8.2)$ & $221(2.2)$ & $3.2(2.4$ to 4.4$)$ \\
\hline Diuretics & $168(18.1)$ & $597(6.1)$ & 3.3 (2.7 to 4.1$)$ \\
\hline Calcium channel blockers & $87(9.4)$ & $604(6.1)$ & $1.4(1.1$ to 1.7$)$ \\
\hline$\beta$-blockers & $141(15.2)$ & $1082(11.0)$ & $1.4(1.1$ to 1.7$)$ \\
\hline
\end{tabular}

(70.0 vs 71.4 years, male sex $33 \% \quad$ vs $63 \% \quad(p=0.6)$, respectively).

\section{Association between use of AEMs and SCD}

Among cases, $23(2.5 \%)$ were current AEM users at the SCD date, and 24 (2.6\%) were past users (table 2). Current AEM use was associated with a 2.6-fold increased SCD risk (cases $2.5 \%$,

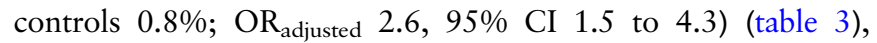
while past use was not (cases 2.6\%, controls $1.7 \%$; OR adjusted 1.4, 95\% CI 0.9 to 2.3) (table 3). Current AEM use was associated with increased SCD risk among symptomatic epilepsy

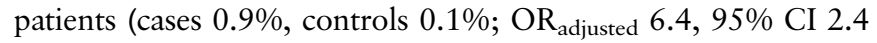

to 17.4 ) (table 3 ) and non-epilepsy patients (cases 1.0\%, controls $0.4 \%$; $\mathrm{OR}_{\text {adjusted }} 2.3,95 \%$ CI 1.01 to 5.2 ) (table 3), but not among stable epilepsy patients (cases $0.7 \%$, controls $0.4 \%$; $\mathrm{OR}_{\text {adjusted }} 1.6,95 \%$ CI 0.7 to 4.1) (table 3). All cases were on monotherapy, except for three who used two AEMs (two patients with symptomatic epilepsy were on carbamazepine/ phenytoin and carbamazepine/valproic acid; one patient with stable patient was on carbamazepine/clonazepam). Patients who had used AEMs for $>30$ days had a higher risk for SCD (cases 2.3\%, controls $0.8 \%$; OR adjusted 2.6 , 95\% CI 1.1 to 6.0 ) (table 4) than those who had used AEMs for $\leq 30$ days (cases $0.2 \%$, controls $0.06 \%$; $\mathrm{OR}_{\text {adjusted }} 0.9,95 \%$ CI 0.1 to 10.7 ) (table 4).

Stratified analyses among current users showed that men (cases

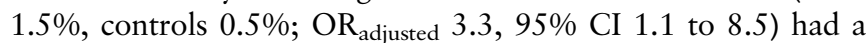
higher risk than women (cases $1.0 \%$, controls $0.3 \%$; OR adjusted $1.4,95 \%$ CI 0.3 to 6.0 ) (table 4 ). This difference was not statistically significant. SCD risk in patients $<65$ years of age (cases $0.8 \%$, controls $0.3 \%$; OR adjusted $2.7,95 \%$ CI 0.6 to 11.2 ) was not significantly different from those $\geq 65$ years old (cases $1.7 \%$, controls $0.6 \%$; OR adjusted $1.2,95 \%$ CI 0.3 to 4.3 ) (table 4).

\section{Association between sodium channel blocking AEMs and SCD}

The highest SCD risk was observed among users of sodium channel blocking AEMs (cases 1.6\%, controls $0.4 \%$; $\mathrm{OR}_{\text {adjusted }}$ 3.0, 95\% CI 1.7 to 5.3) (table 5). The association remained unchanged after correction for epilepsy (cases 1.6\%, controls

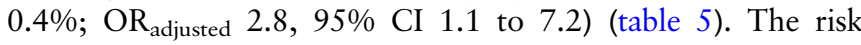
among users of non-sodium channel blocking AEMs was lower and not significant (cases $1.1 \%$, controls $0.5 \%$; OR adjusted 1.7 , $95 \%$ CI 0.5 to 5.3) (table 5). Use of sodium channel blocking AEMs was comparable among epilepsy and non-epilepsy patients (stable vs symptomatic epilepsy: $43 \%$ vs $20 \%,(p=0.6)$; epilepsy vs non-epilepsy: $29 \%$ vs $44 \%(p=0.7))$.

Analyses of individual AEMs showed a statistically significantly increased SCD risk among carbamazepine users (cases

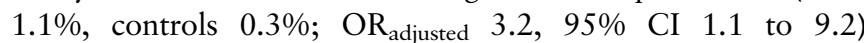
(table 5) and gabapentin users (cases 0.3\%, controls $0.06 \%$; $\mathrm{OR}_{\text {adjusted }} 5.7,95 \%$ CI 1.2 to 27.9) (table 5), also after correction for epilepsy.

\section{DISCUSSION}

We found evidence that both epilepsy and AEM use were associated with increased SCD risk. Symptomatic epilepsy, but not stable epilepsy, was associated with increased SCD risk. AEM use was independently associated with increased SCD risk in epilepsy. SCD risk was also elevated in non-epilepsy patients

Table 2 Epilepsy and risk for SCD

\begin{tabular}{|c|c|c|c|c|}
\hline & $\begin{array}{l}\text { SCD cases } \\
n=926\end{array}$ & $\begin{array}{l}\text { Controls } \\
n=9832\end{array}$ & $\mathrm{OR}^{*}(95 \% \mathrm{Cl})$ & $\mathrm{ORt}(95 \% \mathrm{Cl})$ \\
\hline No epilepsy & $912(98.4)$ & $9783(99.5)$ & 1.0 (reference) & 1.0 (reference) \\
\hline Epilepsył & $14(1.5)$ & $49(0.5)$ & 2.7 (1.4 to 5.3$)$ & $2.8(1.4$ to 5.3$)$ \\
\hline Stable epilepsy§ & $6(0.6)$ & $37(0.4)$ & $1.7(0.7$ to 4.2$)$ & $1.6(0.7$ to 4.1$)$ \\
\hline Symptomatic epilepsyๆ & $8(0.9)$ & $12(0.1)$ & 5.9 (2.1 to 16.1$)$ & 5.8 (2.1 to 15.6$)$ \\
\hline \multicolumn{5}{|c|}{$\begin{array}{l}\text { Data are expressed as number (\%) unless otherwise indicated. *ORs matched for age, gender, practice, and calendar time. } \\
\text { tORs matched for age, gender, practice, and calendar time, adjusted for heart failure. } \\
\text { ¥Patients with a diagnosis of epilepsy and use of AEMs. } \\
\text { §Patients with a diagnosis of epilepsy, use of AEMs, and no seizure in the } 2 \text { years before SCD date. } \\
\text { ๆPatients with a diagnosis of epilepsy, use of AEMs, and seizure in the } 2 \text { years before SCD date. } \\
\text { AEM, antiepileptic medication; SCD, sudden cardiac death. }\end{array}$} \\
\hline
\end{tabular}


Table 3 Use of AEMs and SCD risk

\begin{tabular}{|c|c|c|c|c|}
\hline & $\begin{array}{l}\text { SCD cases } \\
\mathrm{n}=926\end{array}$ & $\begin{array}{l}\text { Controls } \\
n=9832\end{array}$ & $O R^{*}(95 \% \mathrm{Cl})$ & ORt $(95 \% \mathrm{Cl})$ \\
\hline \multicolumn{5}{|l|}{ Overall use } \\
\hline Never use & $879(94.9)$ & $9583(97.5)$ & 1.0 (reference) & 1.0 (reference) \\
\hline Past use & $24(2.6)$ & $166(1.7)$ & 1.5 (1.0 to 2.4$)$ & $1.4(0.9$ to 2.3$)$ \\
\hline Current use & $23(2.5)$ & $83(0.8)$ & 2.8 (1.7 to 4.6$)$ & 2.6 (1.5 to 4.3$)$ \\
\hline \multicolumn{5}{|l|}{ Use for epilepsy } \\
\hline Current use & $14(1.5)$ & $49(0.5)$ & 2.7 (1.4 to 5.3$)$ & $2.8(1.4$ to 5.3$)$ \\
\hline \multicolumn{5}{|c|}{ Use for stable epilepsy } \\
\hline Current use & $6(0.7)$ & $37(0.4)$ & 1.7 (0.7 to 4.1$)$ & $1.6(0.7$ to 4.1$)$ \\
\hline \multicolumn{5}{|c|}{ Use for symptomatic epilepsy } \\
\hline Current use & $8(0.9)$ & $12(0.1)$ & $6.5(2.4$ to 17.5$)$ & $6.4(2.4$ to 17.4$)$ \\
\hline \multicolumn{5}{|c|}{ Use by non-epilepsy patients } \\
\hline Current use & $9(1.0)$ & $34(0.4)$ & 2.7 (1.3 to 5.9$)$ & 2.3 (1.01 to 5.2$)$ \\
\hline
\end{tabular}

who used AEMs for chronic pain syndromes. Sodium channel blocking AEMs were associated with increased SCD risk, whereas AEMs without sodium channel blocking properties were not, indicating that sodium channel blockage may contribute to increased SCD risk in AEM users.

\section{Epilepsy is associated with SCD}

We recently provided the first evidence from a systematic ECG based study that epilepsy is associated with a threefold increased risk for VF and SCD in the general population. ${ }^{8}$ In the present study, performed in a different cohort from the general population, we found a similar threefold increase in SCD risk, confirming this association. The point prevalence of epilepsy in the control group in our present study was $0.5 \%$. This agrees well with the prevalence of epilepsy in the general population reported by us and others, suggesting that we captured all people with epilepsy in our study. ${ }^{8} 23$
We aimed to identify the causes for the association between epilepsy and SCD risk, focusing on the roles of epilepsy per se (possibly mediated by a shared biologic basis of epilepsy and VF, eg, aberrant ion channels) and AEMs (possibly mediated by their cardiac sodium channel blocking properties). ${ }^{24}$ Providing supportive evidence for the role of epilepsy per se, we found that SCD risk was elevated in patients with symptomatic epilepsy, but not stable epilepsy. Thus, a history of recent seizures (in our study, resulting from therapy resistant seizures in almost $90 \%$ of cases) is associated with increased SCD risk. It has been suggested that these uncontrolled seizures in therapy resistant patients, particularly tonic-clonic seizures, might trigger sudden death in epilepsy. This view is derived from reports of sudden unexpected death in epilepsy (SUDEP) cases, but such cases are rarely witnessed. Almost none of the witnessed patients in the present study $(0 \%)$ and our previous ${ }^{8}$ study (8\%) experienced a seizure directly before SCD.

Table 4 Effect modification of SCD risk in users of AEMs

\begin{tabular}{|c|c|c|c|c|c|}
\hline & $\begin{array}{l}\text { SCD cases } \\
\mathrm{n}=926\end{array}$ & $\begin{array}{l}\text { Controls } \\
n=9832\end{array}$ & $\mathrm{OR}^{*}(95 \% \mathrm{Cl})$ & ORt $(95 \% \mathrm{Cl})$ & OR‡ (95\% Cl) \\
\hline Never used & $879(94.9)$ & $9583(97.5)$ & 1.0 (reference) & 1.0 (reference) & 1.0 (reference) \\
\hline Past use & $24(2.6)$ & $166(1.7)$ & 1.5 (1.0 to 2.4$)$ & $1.4(0.9$ to 2.3$)$ & 1.4 (0.9 to 2.2$)$ \\
\hline Current use & $23(2.5)$ & $83(0.8)$ & 2.8 (1.7 to 4.6$)$ & 2.5 (1.5 to 4.2$)$ & 2.3 (1.4 to 3.9 ) \\
\hline \multicolumn{6}{|l|}{ Duration of use (days) } \\
\hline$\leq 30$ & $2(0.2)$ & $6(0.06)$ & $2.6(0.5$ to 14.3$)$ & $1.8(0.3$ to 11.0$)$ & $0.9(0.1$ to 10.7$)$ \\
\hline$>30$ & $21(2.3)$ & $77(0.8)$ & 2.7 (1.6 to 4.6$)$ & 2.6 (1.5 to 4.5$)$ & $2.6(1.1$ to 6.0$)$ \\
\hline \multicolumn{6}{|l|}{ Effect modification } \\
\hline \multicolumn{6}{|l|}{ Gender§ } \\
\hline Current use in females & $9(1.0)$ & $34(0.3)$ & 2.7 (1.2 to 6.1$)$ & 2.7 (1.2 to 6.1$)$ & $1.4(0.3$ to 6.0$)$ \\
\hline Current use in males & $14(1.5)$ & $49(0.5)$ & 2.8 (1.5 to 5.3$)$ & 2.5 (1.3 to 4.8$)$ & 3.3 (1.1 to 8.5$)$ \\
\hline \multicolumn{6}{|l|}{ Age (years) } \\
\hline Current use in $<65$ & $7(0.8)$ & $27(0.3)$ & 4.4 (1.9 to 10.4$)$ & 3.3 (1.3 to 8.4$)$ & 2.7 (0.6 to 11.2$)$ \\
\hline Current use in $\geq 65$ & $16(1.7)$ & $56(0.6)$ & 2.2 (1.2 to 4.1$)$ & $2.2(1.1$ to 4.0$)$ & $1.2(0.3$ to 4.3$)$ \\
\hline \multicolumn{6}{|c|}{$\begin{array}{l}\text { Data are expressed as number (\%) unless otherwise indicated. } \\
\text { *ORs matched for age, gender, practice, and calendar time. } \\
\text { tORs matched for age, gender, practice, and calendar time, adjusted for heart failure. } \\
\text { †ORs matched for age, gender, practice, and calendar time, adjusted for heart failure and epilepsy. } \\
\text { §There was no gender interaction among users of antiepileptic medications ( } p=0.88 \text { ). } \\
\text { AEM, antiepileptic medication; SCD, sudden cardiac death. }\end{array}$} \\
\hline
\end{tabular}


Table 5 Current use of individual AEMs and SCD risk

\begin{tabular}{|c|c|c|c|c|c|}
\hline & $\begin{array}{l}\text { SCD cases } \\
\mathrm{n}=926\end{array}$ & $\begin{array}{l}\text { Controls } \\
\mathrm{n}=9832\end{array}$ & $\mathrm{OR}^{*}(95 \% \mathrm{Cl})$ & ORt $(95 \% \mathrm{Cl})$ & OR‡ (95\% Cl) \\
\hline Sodium channel blocking AEMs§ & $15(1.6)$ & $43(0.4)$ & $3.0(1.7$ to 5.4$)$ & $3.0(1.7$ to 5.3$)$ & 2.8 (1.1 to 7.2$)$ \\
\hline Gabapentin & $3(0.3)$ & $6(0.06)$ & 5.1 (1.1 to 22.3 ) & 5.9 (1.2 to 28.5$)$ & 5.7 (1.2 to 27.9$)$ \\
\hline Lamotrigine & 0 & $3(0.0)$ & NA & NA & NA \\
\hline Oxcarbazepine & 0 & $1(0.0)$ & NA & NA & NA \\
\hline Clonazepam & $4(0.4)$ & $14(0.1)$ & 2.9 (0.9 to 9.4$)$ & 2.1 (0.6 to 7.3 ) & 2.1 (0.6 to 7.2 ) \\
\hline Levetiracetam & 0 & $1(0.0)$ & NA & NA & NA \\
\hline Phenobarbital & $2(0.2)$ & $14(0.1)$ & NA & NA & NA \\
\hline Primidone & 0 & $4(0.0)$ & NA & NA & NA \\
\hline Valproic acid & $4(0.4)$ & $20(0.2)$ & 2.2 (0.7 to 6.8$)$ & $2.0(0.6$ to 6.2$)$ & $1.8(0.4$ to 7.3$)$ \\
\hline
\end{tabular}

Thus, although tonic-clonic seizures are suggested as being direct triggers for sudden death in epilepsy, especially SUDEP, we believe that this is not the case for SCD in epilepsy-that is, sudden death with a cardiac cause. This notion is supported by a recent study which demonstrated that the majority of witnessed SCD in epilepsy patients did not occur in the setting of a tonic-clonic seizure. ${ }^{25}$ Nonetheless, a tonic-clonic seizure may still be a risk factor for SUDEP, due to respiratory causes.

A possible mechanism underlying the susceptibility for SCD in epilepsy might be a shared genetic cause, as suggested in various reports. $^{24}{ }^{26-28}$ In both the brain and the heart, ion channels play a critical role in the generation and conduction of electrical activity. Various ion channels are expressed both in the brain and in the heart, albeit at different levels. ${ }^{29}$ Mutations in genes encoding these ion channels may lead to defective ion channel function and pathological electrical activity, manifesting as epilepsy in the brain and arrhythmia in the heart. Examples of such genes are: (1) KCNQ1, ${ }^{26} 27$ the most common long QT syndrome-causing gene, encoding a potassium channel; (2) $S C N 1 A,{ }^{24} 28$ the gene which encodes the neuronal sodium channel; and (3) SCN $5 A,{ }^{29}$ the most common Brugada syndrome-causing gene, encoding the cardiac sodium channel. As epilepsy patients are at risk for both epileptic seizures and cardiac ventricular arrhythmias, it becomes even more difficult to establish the true cause for seizures in these patients. Epilepsy may cause seizures, but (self-terminating) ventricular tachycardias may also induce hypoxaemia related seizures that mimic epilepsy seizures. Moreover, ventricular tachycardias may not be self-terminating and convert to $\mathrm{VF}$, thereby causing hypoxaemia related seizures and SCD. In these patients, both the seizures and SCD are not linked to epilepsy.

\section{Use of AEMs is associated with SCD}

A possible role for AEMs in SCD has so far been suggested in small cohorts of epilepsy patients with severe epilepsy who were treated at specialised epilepsy institutions, and often used multiple AEMs. ${ }^{30}$ We investigated the role of AEMs in SCD in the general population, assessing SCD risk in stable and symptomatic epilepsy patients (to reduce confounding by disease severity), and in non-epilepsy patients (to exclude confounding by epilepsy). Most patients (87\%) used a single AEM, thereby reducing confounding by polytherapy of AEMs. AEM use was associated with increased SCD risk among symptomatic epilepsy patients and non-epilepsy patients, but not among stable epilepsy patients. In stable patients, a possible proarrhythmic effect of the AEMs may have been outweighed by reduction in sudden death risk by the fact that the epilepsy was well controlled. Importantly, non-epilepsy patients who used AEMs had increased SCD risk. This may be of concern, as the indications for AEM prescriptions (especially carbamazepine and gabapentin) have widened to include chronic pain syndromes.

To investigate further the role of AEMs in SCD, AEMs with sodium channel blocking properties were tested in detail. Previous studies have linked cardiac drugs with sodium channel blocking properties to SCD. ${ }^{9}$ Moreover, patients with an inherited reduction in cardiac sodium current (eg, Brugada syndrome) have an increased risk for VF and SCD. Here, we showed that the use of AEMs with sodium channel blocking properties was associated with an almost threefold increased SCD risk, even after correction for confounding factors and epilepsy. Conversely, AEMs without sodium channel blocking properties exhibited a weaker and non-significant association with SCD. We found carbamazepine and gabapentin, both sodium channel blockers, to be associated with increased SCD risk.

\section{Strength and limitations of the study}

The major strength of our study is its population based design, minimising selection bias. This enabled us to study symptomatic and asymptomatic epilepsy patients. Furthermore, we were able to study patients who used AEMs for various indications. Our access to the databases of GPs allowed us to collect extensive information on drug use, concomitant diseases, potential confounders, and circumstances surrounding death. However, our study also has some limitations. First, although GPs register death consistently, we may have missed some sudden deaths. Secondly, misclassifications of deaths may have occurred. To reduce misclassification, we only included cases with sufficient and clear information on the circumstances surrounding death. Finally, while GP records were accurate in registering epilepsy 
diagnosis and seizures, little additional information on epilepsy type and seizure type was available.

\section{Key messages}

What is already known on this subject?

Epilepsy is associated with sudden death. Recently, epilepsy has also been associated with sudden cardiac death (SCD).

\section{What might this study add?}

Epilepsy is associated with a 2.8-fold increase in SCD risk, and use of antiepileptic medications (AEMs) is associated with a 2.6-fold increase in SCD risk. SCD risk is mediated by poor seizure control and use of AEMs that block cardiac sodium channels.

How might this impact on clinical practice? Seizure control in epilepsy patients may reduce SCD risk. AEMs must be chosen by taking sudden cardiac death risk into account.

Acknowledgements The authors greatly appreciate the contributions of Ann M Vanrolleghem in the coordination of the IPCI data center.

Contributors All authors were involved in substantial contributions to the conception or design of the work; or the acquisition, analysis, or interpretation of data for the work; drafting the work or revising it critically for important intellectual content; final approval of the version to be published; agreement to be accountable for all aspects of the work in ensuring that questions related to the accuracy or integrity of any part of the work are appropriately investigated and resolved.

Funding HLT was supported by the Netherlands Organization for Scientific Research (NWO, grant ZonMW Vici 918.86.616), the Dutch Medicines Evaluation Board (MEB/CBG), the European Community's Seventh Framework Programme (FP7, grant 241679, ARITMO), Biobanking and Biomolecular Research Infrastructure The Netherlands (BBMRI-NL), and the National Epilepsy Foundation Netherlands (project number 10-07). The Arrest data collection was supported by an unconditional grant from Physio Control and a grant from the Netherlands Heart Foundation (grant 2006B 179). AB was supported by the Netherlands Organization for Scientific Research (NWO, grant Mozaiek 017.003.084). MCJMS coordinates the IPCI database, which has received unrestricted research grants from various pharmaceutical companies, including Pfizer, Merck, Johnson \& Johnson, Altana, AstraZeneca, Boehringer, Eli Lilly, and GlaxoSmithKline. KMV received unconditional grants from Yamanouchi, Pfizer, Boehringer Ingelheim, and Novartis. None of these related to the topic of this work.

Competing interests None. All the grants used for this study were unrestricted grants. The funders were not involved in the design and conduct of the study, the collection, management, analysis, and interpretation of the data, or the preparation, review or approval of the manuscript.

Ethics approval The Scientific and Ethical Advisory Board of the IPCI project approved this study.

Provenance and peer review Not commissioned; externally peer reviewed.

\section{REFERENCES}

1 Myerburg RJ, Castellanos A. Cardiac arrest and sudden cardiac death. In: Libby P, Bonow RO, Mann DL, Zipes DP, eds. Braunwald's heart disease: a textbook of cardiovascular medicine. Oxford: Elsevier, 2007: 933-74.

2 Huikuri HV, Castellanos A, Myerburg RJ. Sudden death due to cardiac arrhythmias. N Engl J Med 2001;345:1473-82.
3 Roden DM. Drug-induced prolongation of the QT interval. N Engl J Med 2004;350:1013-22.

4 Bardai A, Amin AS, Blom MT, et al. Sudden cardiac arrest associated with use of a non-cardiac drug that reduces cardiac excitability: evidence from bench, bedside, and community. Eur Heart J 2013;34:1506-16.

5 Postema PG, Wolpert C, Amin AS, et al. Drugs and Brugada syndrome patients: review of the literature, recommendations, and an up-to-date website (http://www. brugadadrugs.org). Heart Rhythm 2009;6:1335-41.

6 Devinsky O. Sudden, unexpected death in epilepsy. N Eng/ J Med 2011;365:1801-11.

7 Surges R, Thijs RD, Tan HL, et al. Sudden unexpected death in epilepsy: risk factors and potential pathomechanisms. Nat Rev Neurol 2009;5:492-504.

8 Bardai A, Lamberts RJ, Blom MT, et al. Epilepsy is a risk factor for sudden cardiac arrest in the general population. PLOS ONE 2012;7:e42749.

9 Greenberg HM, Dwyer EM Jr, Hochman JS, et al. Interaction of ischaemia and encainide/flecainide treatment: a proposed mechanism for the increased mortality in CAST I. Br Heart J 1995;74:631-5.

10 Vlug $A E$, van der Lei J, Mosseveld BM, et al. Postmarketing surveillance based on electronic patient records: the IPCI project. Methods Inf Med 1999;38:339-44.

11 van der Lei J, Duisterhout JS, Westerhof HP, et al. The introduction of computer-based patient records in The Netherlands. Ann Intern Med 1993;119:1036-41.

12 Lamberts $\mathrm{H}$, Wood M, Hofmans-Okkes IM. International primary care classifications: the effect of fifteen years of evolution. Fam Pract 1992;9:330-9.

13 Priori SG, Aliot E, Blomstrom-Lundqvist C, et al. Task force on sudden cardiac death of the European Society of Cardiology. Eur Heart J 2001;22:1374-450.

14 Remy S, Beck H. Molecular and cellular mechanisms of pharmacoresistance in epilepsy. Brain 2006;129:18-35.

15 Ragsdale DS, Avoli M. Sodium channels as molecular targets for antiepileptic drugs. Brain Res 1998;26:16-28.

16 Liu Y, Qin N, Reitz T, et al. Inhibition of the rat brain sodium channel Nav1.2 after prolonged exposure to gabapentin. Epilepsy Res 2006;70:263-8.

17 Johannessen CU, Johannessen SI. Valproate: past, present, and future. CNS Drug Rev 2003;9:199-216.

18 Costa C, Martella G, Picconi B, et al. Multiple mechanisms underlying the neuroprotective effects of antiepileptic drugs against in vitro ischemia. Stroke 2006;37:1319-26.

19 Johannessen CU, Petersen D, Fonnum F, et al. The acute effect of valproate on cerebral energy metabolism in mice. Epilepsy Res 2001;47:247-56.

20 Fisher RS, van Emde Boas W, Blume W, et al. Epileptic seizures and epilepsy: definitions proposed by the International League Against Epilepsy (ILAE) and the International Bureau for Epilepsy (IBE). Epilepsia 2005;46:470-2.

21 WHO. World Health Organization-International Society of Hypertension guidelines for the management of hypertension. Guidelines Subcommittee. J Hypertens 1999;17:151-83.

22 Greenland S. Modeling and variable selection in epidemiologic analysis. Am J Public Health 1989;79:340-9.

23 Forsgren L, Beghi E, Oun A, et al. The epidemiology of epilepsy in Europe-a systematic review. Eur J Neurol 2005;12:245-53.

24 Kalume F, Westenbroek RE, Cheah CS, et al. Sudden unexpected death in a mouse model of Dravet syndrome. J Clin Invest 2013;123:1798-808.

25 Stecker EC, Reinier K, Uy-Evanado A, et al. Relationship between seizure episode and sudden cardiac arrest in patients with epilepsy: a community-based study. Circ Arrhythm Electrophysiol 2013;6:912-16.

26 Goldman AM, Glasscock E, Yoo J, et al. Arrhythmia in heart and brain: KCNQ1 mutations link epilepsy and sudden unexplained death. Sci Trans/ Med 2009;1:2ra6.

27 Johnson JN, Hofman N, Haglund CM, et al. Identification of a possible pathogenic link between congenital long QT syndrome and epilepsy. Neurology 2009;72:224-31.

28 Klassen TL, Bomben VC, Patel A, et al. High-resolution molecular genomic autopsy reveals complex sudden unexpected death in epilepsy risk profile. Epilepsia 2014;55:e6-12.

29 Haufe V, Cordeiro JM, Zimmer T, et al. Contribution of neuronal sodium channels to the cardiac fast sodium current INa is greater in dog heart Purkinje fibers than in ventricles. Cardiovasc Res 2005;65:117-27.

30 Timmings PL. Sudden unexpected death in epilepsy: is carbamazepine implicated? Seizure 1998;7:289-91. 